This document is the accepted manuscript version of the following article:

C. Wäckerlin, K. Tarafder, D. Siewert, J. Girovsky, T. Hählen, C. Iacovita, A. Kleibert, F. Nolting, T.

A. Jung, P. M. Oppeneer, N. Ballav

On-surface coordination chemistry of planar molecular spin systems: novel magnetochemical effects induced by axial ligands

Chemical Science, 2012, 3, 3154-3160, https://dx.doi.org/10.1039/c2sc20828h

\title{
On-surface coordination chemistry of planar molecular spin systems: Novel magnetochemical effects induced by axial ligands
}

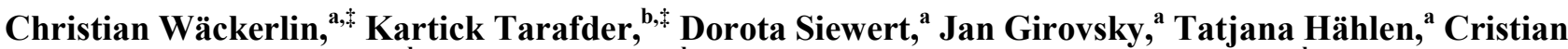 \\ Iacovita, ${ }^{c}$ Armin Kleibert, ${ }^{d}$ Frithjof Nolting, ${ }^{d}$ Thomas A. Jung, ${ }^{a, *}$ Peter M. Oppeneer, ${ }^{b, *}$ and Nirmalya \\ s Ballav, ${ }^{\mathrm{e}, *}$
}

\section{Received (in XXX, XXX) Xth XXXXXXXXX 20XX, Accepted Xth XXXXXXXXX 20XX}

Paramagnetic transition-metal complexes assembled on surfaces are of great interest for potential applications in organic spintronics. The magnetochemical interactions of the spin of the metal centers with both ferromagnetic surfaces and optional axial ligands are yet to be understood. We use a

10 combination of X-ray magnetic circular dichroism (XMCD) and quantum-chemical simulations based on density functional theory $(\mathrm{DFT}+\mathrm{U})$ to investigate these metal-organic interfaces with chemically tunable magnetization. The interplay between an optional axial ligand ( $\mathrm{NO}$, spin $\mathrm{S}=1 / 2 \mathrm{or} \mathrm{NH}_{3}, \mathrm{~S}=0$ ) and $\mathrm{Ni}$ and Co ferromagnetic surfaces affecting the spin of $\mathrm{Co}(\mathrm{II})$ tetraphenylporphyrin $\left(\mathrm{d}^{7}, \mathrm{~S}=1 / 2\right), \mathrm{Fe}$ (II)

tetraphenylporphyrin ( $\left.d^{6}, S=1\right), M n(I I)$ tetraphenylporphyrin $\left(d^{5}, S=5 / 2\right)$ and $M n(I I)$ phthalocyanine $\left(d^{5}\right.$, ${ }_{15} \mathrm{~S}=3 / 2$ ) is studied. We find that the structural trans effect on the surface rules the molecular spin state as well as the sign and strength of the exchange interaction with the substrate. We refer to this observation as the surface spin-trans effect.

\section{Introduction}

A large portfolio of concepts in coordination chemistry of ${ }_{20}$ porphyrin and phthalocyanine based metallo-organic complexes has been developed to rationalize the broad spectrum of physicochemical functionalities ${ }^{1}$. Recently, coordination chemistry on the surface is being explored ${ }^{2-6}$. In the specific case of competitive coordination which is usually referred to as the trans effect ${ }^{7}$, the

25 ligand on one side of a metallo-organic complex is affecting the ground state energy or the bonding/unbonding kinetics with the second ligand on the opposite side. To understand coordination chemistry with the surface acting as a ligand ${ }^{8-10}$, square-planar complexes and their reaction with axial ligands like NO, CO, ${ }_{30} \mathrm{NH}_{3}$ affecting the molecule-substrate bonding, have been monitored experimentally.

${ }^{a}$ Laboratory for Micro- and Nanotechnology, Paul Scherrer Institut, 5232 Villigen, Switzerland

35 E-mail: thomas.jung@psi.ch

${ }^{b}$ Department of Physics and Astronomy, Uppsala University, Box 516, S75120 Uppsala, Sweden

E-mail:peter.oppeneer@fysik.uu.se

${ }^{c}$ Department of Physics, University of Basel, 4056 Basel, Switzerland ${ }_{40}^{d}$ Swiss Light Source, Paul Scherrer Institut, 5232 Villigen, Switzerland

${ }^{e}$ Department of Chemistry, Indian Institute of Science Education and Research (IISER), Pune - 411008, India

E-mail:nballav@iiserpune.ac.in

$\dagger$ Electronic Supplementary Information (ESI) available: experimental 45 section; additional STM, XAS, XMCD and DFT+U data; evaluation of exchange coupling strength.

$\ddagger$ Contributed equally.
This has been performed by measurements of the spectral 50 characteristics in X-ray photoelectron spectroscopy (XPS) ${ }^{9,11,10}$, in UV photoelectron spectroscopy (UPS) ${ }^{9,10}$ and in scanning tunneling microscopy/spectroscopy (STM/STS) ${ }^{10,6}$. The term surface trans effect has recently been introduced to describe the observed reduction in bonding with the "surface-ligand" 10 .

55 Magnetochemical interactions of spin-bearing, square-planar transition metal complexes like porphyrins and phthalocyanines with ferromagnetic surfaces offer a unique platform to fabricate metal-organic interfaces with stable magnetization at room temperature that are of great interest for organic spintronics. The 60 search for gaining control over the magnetic properties of these interfaces has revealed a number of important findings: paramagnetic complexes on surfaces may be magnetized by their specific bonding interactions with the ferromagnetic (FM) surface $^{12-20}$. Also, the coordination of surface-ligands to ${ }_{65}$ paramagnetic metallo-porphyrins and phthalocyanines often results in a significant hybridization and/or charge transfer ${ }^{21-26}$. Furthermore, it has been demonstrated that the spin state in cobalt porphyrin adsorbed on a ferromagnetic $\mathrm{Ni}$ substrate can be controlled by axial coordination on the open site, e.g. by $\mathrm{NO}^{27}$.

70 The present work aims at answering the fundamentally important question arising from the above findings: to what extent can classical coordination chemistry concepts, which do not consider the surface specific molecule-substrate interaction, be used to understand the on-surface magnetochemistry of metallo-organic 75 complexes?

We show that the effect of axial coordination onto the spin of planar coordination complexes like porphyrins and phthalocyanines supported on FM substrates can only be understood by inclusion of the surface-ligand into the 
considerations. Our studies demonstrate that on-surface axial ligation can lead to i) spin states which are decisively changed by the interaction with the substrate, ii) a change in the spin alignment (sign of the exchange coupling: parallel or anti5 parallel) or iii) a modification of the strength of the exchange coupling. These findings are based on X-ray magnetic circular dichroism (XMCD) spectroscopy and density functional theory calculations with additional Hubbard interaction taken into account $(\mathrm{DFT}+\mathrm{U})$. i) and ii) are revealed in the magnetochemistry 10 of metallo-tetraphenylporphyrins (CoTPP, FeTPP and MnTPP) sandwiched between a FM surface (Co or Ni thin films) and the NO ligand. To demonstrate iii), we investigate the strongly chemisorbed $^{28} \mathrm{Mn}$-phthalocyanine/Co $(\mathrm{MnPc} / \mathrm{Co})$ system under the influence of the non-spin-bearing $\mathrm{NH}_{3}$ ligand in comparison 15 to the spin-bearing NO ligand. Therefore, the on-surface coordination of metallo-organic molecules is shown to lead to novel magnetochemical effects beyond those of classical coordination chemistry. Hence our observations classify as evidence for the surface spin-trans effect. Moreover, our data 20 provide case studies for engineering magnetic metallo-organic interfaces in future spintronic applications.

\section{Results}

\section{Research design}

${ }_{25}$ The electronic and magnetic properties of both the ad-molecules and the substrates are investigated by X-ray absorption spectroscopy (XAS) and XMCD spectroscopy (Fig. 1a). In the latter technique, circularly polarized X-rays from a synchrotron source with opposite helicities are used to perform the absorption 30 experiments $^{29}$. XMCD, the difference of the XAS for opposite helicities, measured at the $\mathrm{L}_{2,3}$-adsorption edges of the $3 \mathrm{~d}$ transition metals provides information on the magnetization of both, the substrates and the adsorbed transition-metal complexes, separately, in an element-specific manner. Our experimental 35 observations are complemented by density functional theory $(\mathrm{DFT}+\mathrm{U})$ calculations in order to reveal the effects of the axial ligation onto the electronic and spin states. In the DFT $+\mathrm{U}$ approach, the strong Coulomb interactions that are present within the open $3 \mathrm{~d}$-shell of the central metal ion are captured by the 40 supplemented Hubbard U and exchange constant J. This approach has been shown to provide the correct spin state for free as well as substrate absorbed metal-porphyrins ${ }^{15,17,30}$. To manage computational efforts, we have carried out numerical calculations of the on-surface metallo-porphins (metal-P), i.e. without phenyl 45 substitution $^{13,31,16,17}$.

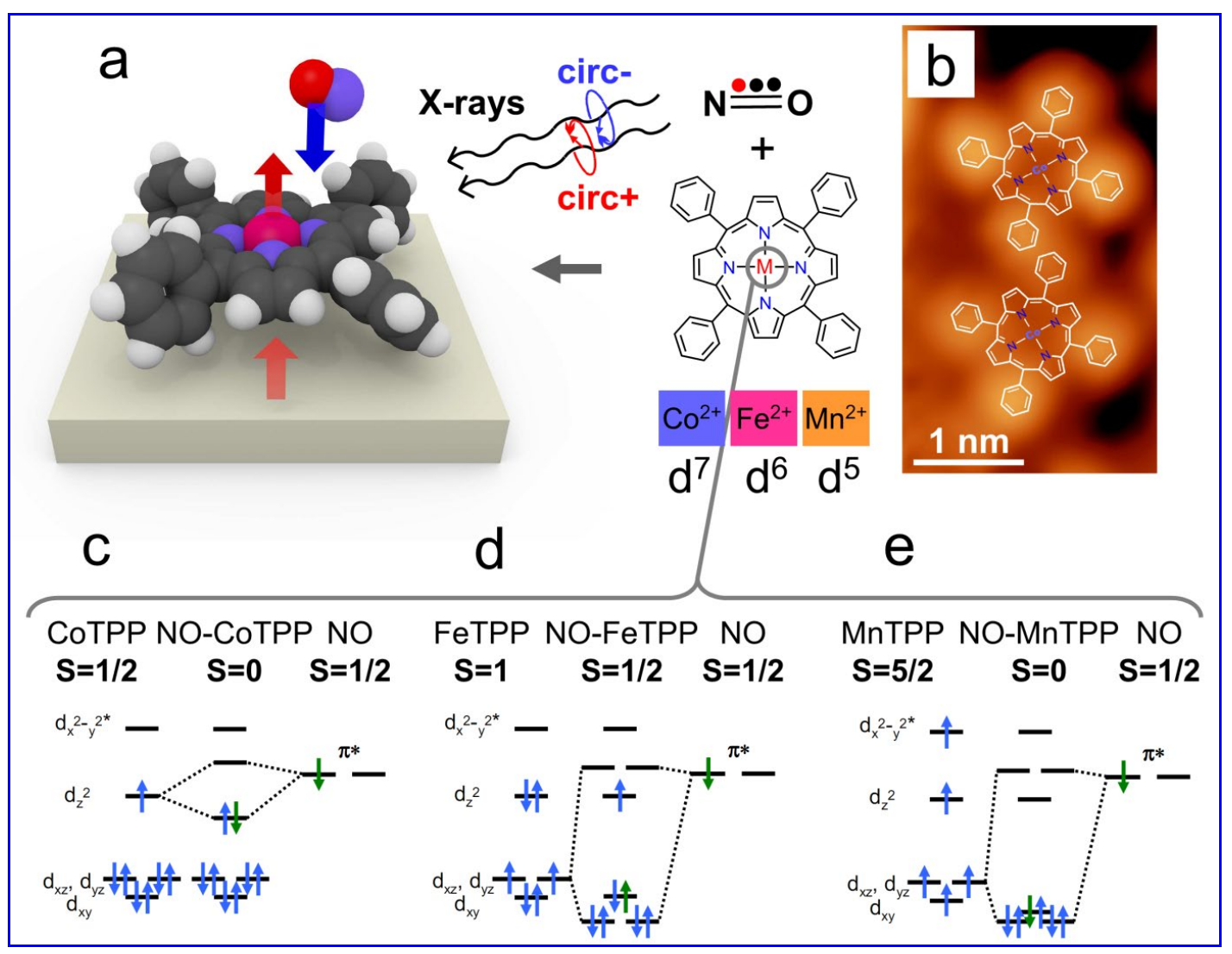

Fig. 1 (a) Schematic representation of the X-ray magnetic circular dichroism (XMCD) experiment on metal-tetraphenylporphyrin (metal-TPP) adsorbed 50 on a ferromagnetic thin film substrate. The magnetic moment of the transition metal centres which is induced by exchange interaction with the substrate is studied by XMCD before and after NO coordination. (b) Scanning tunneling microscopy (STM) of CoTPP on Ni showing that the molecules lie flat on the substrate. As a guide to the eye the chemical schemes have been superimposed in the STM data. (c-e) Molecular orbital (MO) diagrams depicting the reactions of $\mathrm{Co}, \mathrm{Fe}$ and $\mathrm{Mn}$ TPPs with $\mathrm{NO}$ in absence of the surface-ligands. 
In addition, scanning tunneling microscopy (STM) experiments provide insight into the $2 \mathrm{D}$ arrangement of the spin-bearing molecules at a molecular level and into the morphology of the 5 samples in general (Figs. $1 \mathrm{~b}$ and $\mathrm{S} 1$ in the ESI $\dagger$ ). X-ray photoelectron spectroscopy (XPS) is primarily used to monitor the surface composition, i.e. substrate metal film thickness, molecular coverage and stoichiometry.

We have systematically studied the adsorption of $\mathrm{d}^{7}, \mathrm{~d}^{6}$, and $\mathrm{d}^{5}$ 10 transition metallo-porphyrins (Co(II)TPP, Fe(II)TPP and $\mathrm{Mn}$ (II)TPP) onto the FM substrates. The spin of these squareplanar complexes has been investigated with respect to the substrate-molecular bonding and the competitive axial ligation with NO. In the free molecules, the low-spin $(S=1 / 2)^{9}$ and 15 intermediate-spin $(\mathrm{S}=1)^{32}$ and high-spin $(\mathrm{S}=5 / 2)^{33}$ states of CoTPP, FeTPP and MnTPP respectively are reconfigured to low- spin $(\mathrm{S}=0,1 / 2,0)^{9,34-36}$ upon $\mathrm{NO}(\mathrm{S}=1 / 2)$ coordination. As a basis for the discussion of the on-surface coordination in the focus of this paper, molecular orbital (MO) diagrams ${ }^{37}$ illustrating the NO 20 binding in absence of the surface-ligand are provided (Figs. 1c-e). $\mathrm{XMCD}$ experiments performed on the above spin-systems allow us to investigate the complex electronic and spin configurations which arise from the competitive interaction between the surfaceligand and the axial NO ligand. In addition to providing one 25 unpaired electron, NO can undergo a non-innocent electrontransfer reaction ${ }^{38}$. Thus, to assess the influence of the axial coordination onto the strength of the exchange coupling with the surface-ligand, we have chosen to study the strongly chemisorbed $\mathrm{MnPc} / \mathrm{Co}$ system and its response to innocent ${ }^{38}$ and non-spin30 bearing $\mathrm{NH}_{3}(\mathrm{~S}=0)$.

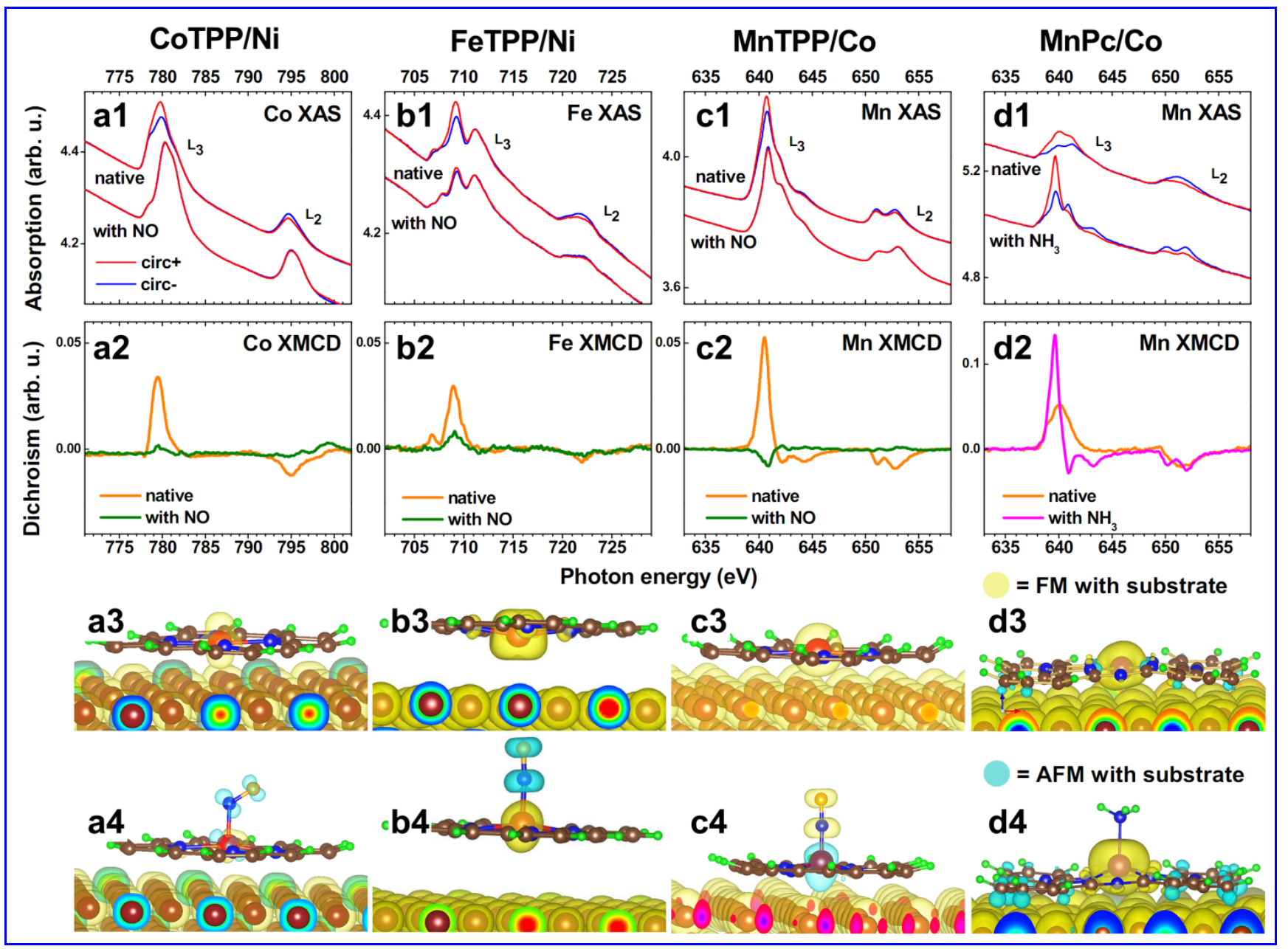

Fig. 2 XAS of CoTPP/Ni (a1), FeTPP/Ni (b1), MnTPP/Co (c1) and MnPc/Co (d1) measured at the respective L-edges, before and after exposure with the 35 gaseous ligand $\left(\mathrm{NO}\right.$ or $\left.\mathrm{NH}_{3}\right)$. The corresponding XMCD spectra (a2-d2), clearly demonstrate an induced magnetic moment in the molecular spin systems. Positive dichroism at the $\mathrm{L}_{3}$ edges corresponds to ferromagnetic exchange coupling with the substrate. NO ligation modifies the XAS peak shape. The $\mathrm{XMCD}$ spectra show the respective magnetic response of the spin bearing molecules: the magnetic moment in CoTPP/Ni is almost completely quenched, for FeTPP/Ni it is modified and reduced while for MnTPP/Co it is also reduced but the sign of the XMCD signal is inverted. This corresponds to an antiferromagnetically coupled magnetic moment. In the case of $\mathrm{MnPc} / \mathrm{Co}$ (at low temperatures), the increased circular dichroism after $\mathrm{NH}_{3}$ coordination 40 indicates an increased spin. The magnetization density isosurface plots from the DFT+U calculation of the respective systems before (a3-d3) and after addition of the ligand (a4-d4) illustrate the spin density distribution and the bonding geometry. The light yellow isosurface denotes spin densities parallel to the substrate (ferromagnetic) and the light blue isosurface denotes spin densities antiparallel (antiferromagnetic) to the substrate spin. 


\section{Cite this: DOI: 10.1039/c0xx00000x}

\section{XAS and XMCD spectroscopy}

Fig. 2 compares the $\mathrm{Co}, \mathrm{Fe}$ and $\mathrm{Mn}_{2,3}$-edges XAS and XMCD spectra recorded on CoTPP/Ni, FeTPP/Ni, MnTPP/Co and $\mathrm{MnPc} / \mathrm{Co}$ systems. In the native state, i.e. before axial ligation, 5 the observed energy positions of the $\mathrm{L}_{3}$-edge XAS signals appear at $\sim 779.8, \sim 709.2, \sim 640.7$ and $\sim 640.0 \mathrm{eV}$, respectively, suggesting considerable electronic interaction of the central metal ion with the axial surface-ligand since the oxidation states of the respective central metal ions seem to be $\leq+2$ in comparison to 10 the molecular bulk states ${ }^{27,13,18,28,39}$. The XMCD signals clearly demonstrate that there is a stable magnetic moment of the admolecule. This magnetic order in the sub-monolayer regime of paramagnetic molecules on FM substrates originates from the considerable exchange interaction between the substrate and the 15 ad-molecule $\mathrm{e}^{7-9,10,13,16}$. From the parallel orientations of the $\mathrm{L}_{2,3^{-}}$ edges XMCD signals of $\mathrm{Co}, \mathrm{Fe}$ and $\mathrm{Mn}$ with respect to those of the substrates (Fig. S2 in the ESI $\dagger$ ), a FM coupling is concluded in all native cases. Angular-dependent XMCD measurements reveal a collinear alignment of the molecular spins with the
20 substrate magnetization ${ }^{13}$. Notably, it is not always the case that the paramagnetic molecule retains its spin upon adsorption; as for example, for $\mathrm{Co}(\mathrm{II})$ phthalocyanine $(\mathrm{CoPc})^{40-42,21,43}$ on $\mathrm{Ni}$ substrate we do not observe an XMCD signal (Fig. S3 in the $\mathrm{ESI} \dagger)$.

${ }_{25} \mathrm{NO}$ coordination leads to almost complete quenching of the $\mathrm{XMCD}$ signal in CoTPP $/ \mathrm{Ni}$, while for $\mathrm{FeTPP} / \mathrm{Ni}$ and for MnTPP/Co the XMCD signal is reduced but still present (Figs. 2a2-c2). Remarkably, the dichroic signal in NO-exposed $\mathrm{MnTPP} / \mathrm{Co}$ is oriented antiparallel with respect to the substrate. 30 Thus, the NO-CoTPP $(\mathrm{S}=0)$ and NO-FeTPP $(\mathrm{S}=1 / 2)$ in the onsurface configuration behave in first approximation as anticipated by MO theory (Figs. 1c and 1d). However, MO theory predicts a spin state $(\mathrm{S}=0)$ for NO-MnTPP (Fig. 1e), which is not seen in our experiment. Moreover, the reversed sign of the circular 35 dichroism of NO-MnTPP, indicates an antiferromagnetic (AFM) coupling which is contrary to the usually observed FM coupling found in experiments on comparable systems ${ }^{12,13,27,28}$.

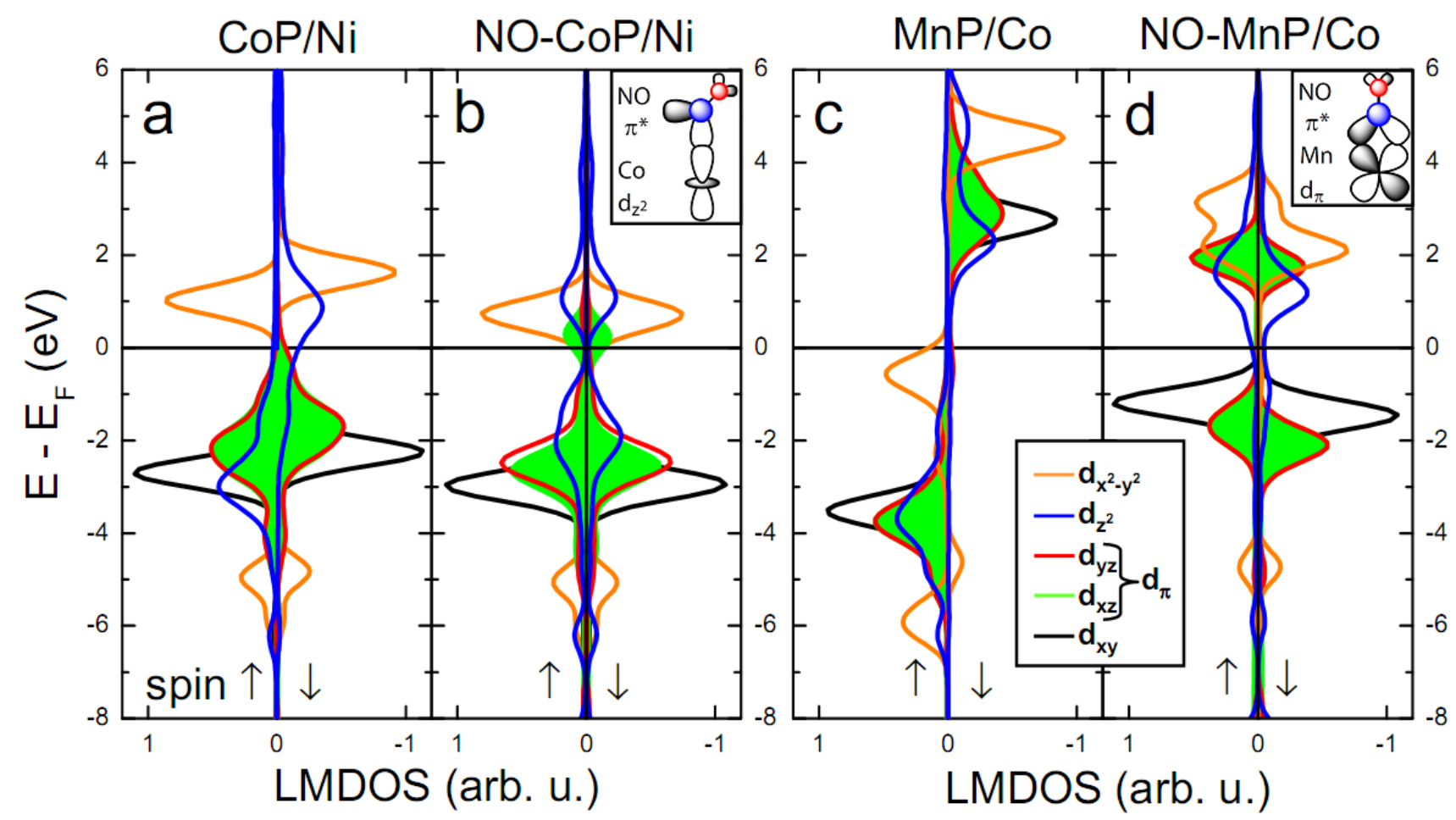

Fig. 3 The $3 \mathrm{~d}$ orbital local magnetic density of states (LMDOS) is shown for CoP/Ni (a), NO-CoP/Ni (b), MnP/Co (c) and NO-MnP/Co (d) in the chemisorbed case. Without NO, both $\mathrm{CoP}$ and $\mathrm{MnP}$ are found to be ferromagnetically (FM) coupled with the respective substrates. The out of plane $\mathrm{d}_{\mathrm{z}}{ }^{2}$ and $\mathrm{d}_{\pi}$ orbitals of $\mathrm{CoP}$ and $\mathrm{MnP}$ are hybridized with the substrate as reflected in the broadening of the respective states. In case of CoP/Ni, the hybridization of $\mathrm{d}_{\mathrm{z}}^{2}$ leads to a reduction of the spin below the initial value of $\mathrm{S}=1 / 2$. The binding of NO onto the surface-supported CoP can be rationalized 45 in the formation of a $\sigma$-bond with $\mathrm{d}_{z}^{2}$ of Co (depicted in the inset) as seen in the separation of the state. In case of MnP, a $\pi$-bond between $\mathrm{d}_{\pi}$ of Mn and $\pi^{*} \square$ of NO is formed as depicted in the inset. The NO binding is found to weaken the hybridization of the $\mathrm{d}$ states with the substrate. The spin state of the on-surface $\mathrm{NO}-\mathrm{CoP}$ is approximately zero $(\mathrm{S} \approx 0)$ in good agreement with considerations neglecting the substrate $(\mathrm{S}=0)$. In contrast, the spin state of the onsurface NO-MnP complex can not be rationalized in neglect of the surface where a spin state of $\mathrm{S}=0$ is expected. Consistent with the experiments, a spin state between $S=1 / 2$ and $S=0$ is found to be antiferromagnetically coupled to the substrate. 
The XAS of the transition metal centers are also significantly modified upon NO exposure (Figs. 2a1-c1): this modification can be primarily related to i) a change in the availability of empty 5 states for the transition of $2 p$ core electrons to the unoccupied $3 d$ levels being excited by the X-ray photons, and/or ii) the change in the oxidation states of the metal ions after ligation with $\mathrm{NO}^{9-11}$. The $\mathrm{Co}, \mathrm{Fe}$ and $\mathrm{Mn} \mathrm{L}_{3}$-edge XAS signals are observed with their maxima at $\sim 780.4, \sim 709.3$ and $\sim 640.9 \mathrm{eV}$, respectively after the $10 \mathrm{NO}$ ligation. The stronger shift of the X-ray absorption peak upon NO coordination for CoTPP $(+0.4 \mathrm{eV})$ compared to NO-FeTPP $(+0.1 \mathrm{eV})$ and NO-MnTPP $(+0.2 \mathrm{eV})$ resembles the versatile coordination chemistry of $\mathrm{NO}$ which is related to the NO-central metal(M) bond angle. The NO-M bond is known to be bent $15\left(\sim 120^{\circ}\right)$ for NO-CoTPP and linear $\left(\sim 180^{\circ}\right)$ in case of NOMnTPP; NO-FeTPP is found to have an intermediate bondangle $^{44}$. In the case of bent M-NO where back-bonding is less important, $\mathrm{NO}$ is sometimes described as the anionic $\pi$-ligand $\left(\mathrm{NO}^{-}\right)$with the tendency to withdraw charge from the metal 20 center whereas with an efficient back-bonding in case of the linear $\left(\sim 180^{\circ}\right)$ bond the increase of the metal oxidation state is smaller. The DFT $+U$ calculations of the on-surface complexes reproduce these bond angles (Figs. 2a4-d4).

A surprisingly different system is given by $\mathrm{MnPc} / \mathrm{Co}$ ${ }_{25}$ (intermediate spin state of $\mathrm{S}=3 / 2$ in absence of the substrate ${ }^{45}$ ) which strongly chemisorbes to the Co substrate ${ }^{28}$. We have studied the strength of the magnetic coupling to the surface before and after $\mathrm{NH}_{3}$ coordination by measuring the dependence of the XMCD/XAS ratio as a function of the temperature. $\mathrm{NH}_{3}$ $30(\mathrm{~S}=0)$ was chosen since NO coordination with the $\mathrm{d}^{5}$ ion in NOMnTPP/Co (Fig. 2c) leads to a low spin state, i.e. a smaller $\mathrm{XMCD}$. For $\mathrm{MnPc} / \mathrm{Co}$ (Figs. 2d1-d2) we find that the $\mathrm{Mn}$ $\mathrm{XMCD} / \mathrm{XAS}$ ratio (Fig. S4 in the $\mathrm{ESI}+$ ), i.e. the magnetization, exhibits almost no temperature dependence in the range of $45-$

$35373 \mathrm{~K}$, thus providing evidence for a strong molecule-substrate magnetic coupling. After coordination with $\mathrm{NH}_{3}$, the $\mathrm{XMCD} / \mathrm{XAS}$ ratio shows a strong decrease when approaching room temperature (Fig. S4 in the ESI $\dagger$ ), thus indicating a significantly weaker exchange coupling. However, while the 40 exchange interaction is reduced, the data recorded at $\sim 80 \mathrm{~K}$ indicate a sizeable increase of the spin after $\mathrm{NH}_{3}$ coordination (Figs. 2d1-d2).

In the following, the experimental results of all four spin-systems are interpreted at a microscopic level by means of DFT $+\mathrm{U}$ 45 calculations, as illustrated in the magnetization density plots (Figs. 2a3-d3 and 2a4-d4). The DFT $+\mathrm{U}$ calculations predict magnetization changes completely consistent with the XMCD measurements. This is further discussed in the next sections, evidencing towards the surface spin-trans effect.

\section{${ }_{50}$ Discussion}

On-surface magnetochemistry in low-spin and high-spin metallo-porphyrins: NO coordination to $\mathrm{CoP} / \mathrm{Ni}$ and $\mathrm{MnP} / \mathrm{Co}$

The mechanism of NO binding with low-spin $\mathrm{d}^{7} \mathrm{Co}(\mathrm{II})$ and highspin $d^{5} \mathrm{Mn}$ (II) porphyrin in the free molecule and the formation 55 of low-spin $(\mathrm{S}=0)$ nitrosyl complexes ${ }^{9,36}$ can be rationalized by the MO diagrams shown earlier (Figs. 1c and 1e). In the low-spin $\mathrm{Co}(\mathrm{II})$ porphyrin, only the singly occupied $\mathrm{d}_{\mathrm{z}}^{2}$ is available for bonding, thus the NO binding results in a $\sigma$-bond with the singly occupied $\pi^{*}$ orbital of NO, whereas in the high-spin $\mathrm{Mn}(\mathrm{II})$ 60 porphyrin the availability of singly occupied $\mathrm{d}_{\pi}$ orbitals (i.e. $\mathrm{d}_{\mathrm{xz}}$ and $\mathrm{d}_{\mathrm{yz}}$ ) results in $\pi$-bonding with $\mathrm{NO}$ - schematically shown in the insets in Fig. 3. Our experimental and theoretical results on these two systems confirm that on the surface, NO binding proceeds in the same fashion. This is reflected in i) the higher ${ }_{65}$ electron affinity of $\sigma$-binding $\mathrm{NO}$ as confirmed in the observed stronger shift upon reaction with NO in Co XAS with respect to Mn XAS, ii) the calculated M-NO bond angles (Figs. 2a4 and $2 \mathrm{c} 4$ ) and iii) the hybridization of the $d$ states with NO (Figs. 3b and $3 \mathrm{~d})$.

${ }_{70}$ Recent DFT $+\mathrm{U}$ calculations showed that metallo-porphyrins can chemisorb or physisorb on metallic substrates ${ }^{31}$. In the case of chemisorbed metal-organic molecules Van der Waals interaction corrections can be neglected. In our $\mathrm{DFT}+\mathrm{U}$ calculations for chemisorbed $\mathrm{CoP} / \mathrm{Ni}$ (Fig. 3a), the hybridization of the singly 75 occupied $\mathrm{d}_{\mathrm{z}}^{2}$ orbital with the substrate yields a reduced spin state of $\sim 0.71 \mu_{\mathrm{B}}$ on Co. For the on surface NO-complex (Fig. 3b) we find a magnetic moment of $\sim 0.04 \mu_{\mathrm{B}}$ on Co, i.e. the spin is almost completely quenched and the local magnetic density of states (LMDOS) is now equally distributed over the two spin-channels 80 (spin $\uparrow$ and spin $\downarrow$ ). The quenching of the spin is in accordance to our experimental results (Figs. 2a1 and 2a2) and similar to the outcome for the free molecule (Fig. 1c). The hybridization of the $\mathrm{d}_{\mathrm{z}}{ }^{2}$ with the substrate is reduced in favor of hybridization with $\mathrm{NO}$ and separation into bonding and antibonding states. Notably, 85 the distance between the Co-ion and the substrate is increased only slightly (by $0.06 \AA$ from initial $2.34 \AA$ ) thus reflecting that NO exerts here only a small structural trans effect ${ }^{7,44}$. Notably, the observed reduction of the magnetic moment due to hybridization with the substrate is consistent with our XMCD 90 data of Co-octaethylporphyrin/ $\mathrm{Ni}(\mathrm{CoOEP} / \mathrm{Ni})$ and $\mathrm{CoPc} / \mathrm{Ni}$ systems, where we find that the magnetic moment is stronger reduced in CoOEP/Ni which has less bulky substituents than CoTPP and is even quenched in case of $\mathrm{CoPc} / \mathrm{Ni}$ (Fig. S2 in the $\mathrm{ESI} \dagger$ ).In contrast to $\mathrm{CoP} / \mathrm{Ni}$, the influence of the surface-ligand 95 onto the spin is clearly visible in $\mathrm{MnP} / \mathrm{Co}$ (Figs. 3c and 3d): without NO, the spin state is slightly reduced to $\sim 4.35 \mu_{\mathrm{B}}$ on $\mathrm{Mn}$ (between $\mathrm{S}=2$ and $5 / 2$, c.f. Ref. 31) NO binding affects all $\mathrm{d}$ states and yields a significant structural trans effect as reflected in the increased distance (by $0.3 \AA$ from initial from $2.11 \AA$ ) 100 between the Mn-ion and the surface. This leads to a decreased hybridization between the substrate and $\mathrm{d}_{\pi}$ (now hybridized with NO and separated into bonding and antibonding states) and of $\mathrm{d}_{\mathrm{z}}^{2}$ (now mostly unoccupied orbitals of $\mathrm{Mn}$ ). Importantly, while reducing the spin significantly towards a low-spin state $(S=0)$, a 105 magnetic moment of $-0.49 \mu_{\mathrm{B}}$ remains AFM coupled with the substrate. In Mn-XMCD (Fig. 2c2), this is expressed by the inverted sign of the circular dichroism.

X-ray diffraction (XRD) data of NO-CoTPP in bulk shows a displacement of the Co ion from the center of $0.09 \AA$, as value 110 which is slightly larger then the calculated Co lift-up on surface 46. The Co-N(NO) bond-length $(1.83 \AA)$ and angle $\left(\leq 128.5^{\circ}\right)$ correspond very well with the calculated on-surface bond-length $(1.82 \AA)$ and angle $\left(\sim 123.6^{\circ}\right)$. In case of NO-MnTPP in bulk, XRD data shows a Mn-N(NO) bond length of $1.64 \AA$ and a linear $115\left(177.8^{\circ}\right) \mathrm{Mn}-\mathrm{N}-\mathrm{O}$ bond ${ }^{47}$. This corresponds very well with the 
calculated on-surface Mn-N(NO) bond length (1.63 $\AA)$ and angle $\left(177.0^{\circ}\right)$. The displacement of the Mn ion from the center $(0.34$ $\AA)$ is comparable to calculated value for the on-surface configuration. Overall, the structural characteristics of the 5 calculated on-surface $\mathrm{Co}, \mathrm{Fe}$ and $\mathrm{Mn}$ porphyrines correspond very closely with the XRD data of the bulk species. However, this is not the case for the spin states, the most remarkable feature being provided by the AFM coupled spin in the on-surface NOMnTPP complex.

10

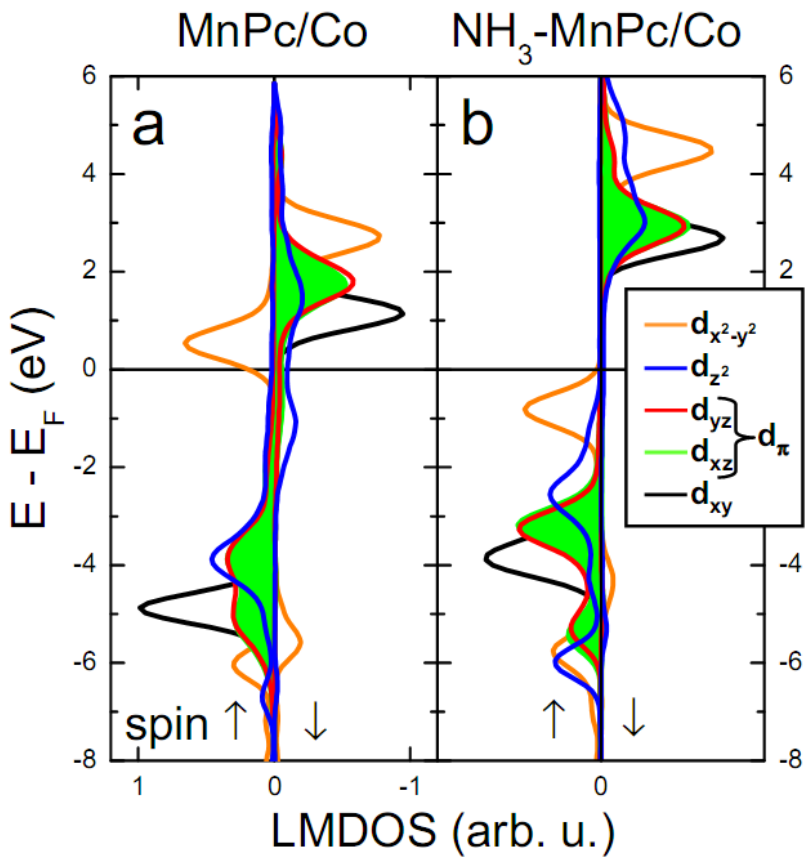

Fig. 4 The 3d LMDOS for $\mathrm{MnPc} / \mathrm{Co}$ (a) and $\mathrm{NH}_{3}-\mathrm{MnPc} / \mathrm{Co}$ (b), in the chemisorbed case. Without $\mathrm{NH}_{3}$, the out of plane $\mathrm{d}_{\mathrm{z}}{ }^{2}$ and $\mathrm{d}_{\pi}$ orbitals are significantly hybridized with the substrate as recognized by their 15 broadening. In the $\mathrm{NH}_{3}$ complex, the hybridization with the substrate is weakened providing narrower d-states. In the calculated on-surface structure the $\mathrm{Mn}$-ion is pulled out of the plane towards the $\mathrm{NH}_{3}$ (Figure $2 \mathrm{~d} 4)$. The spin state of $\mathrm{Mn}$ is increased from approximately intermediate spin to approximately high-spin. This is experimentally observed in the 20 increased XMCD/XAS ratio at low temperatures (Figure S4 in the ESI $†$ ). The weaker hybridization with the substrate is reflected in a strong reduction of the calculated magnetic coupling strength. This is confirmed by a stronger decrease of the XMCD/XAS ratio of the $\mathrm{NH}_{3}$-complex in our experiments performed with increasingly higher temperature. A 25 ferromagnetic coupling is found before and after $\mathrm{NH}_{3}$ coordination.

On-surface magnetochemistry (FeP/Ni + NO): Tuning of the molecular spin

In contrast to $\mathrm{d}^{7} \mathrm{Co}$ and $\mathrm{d}^{5} \mathrm{Mn}$ porphyrins where a quenching of the magnetic moment is anticipated, the case of NO coordination 30 with $d^{6} \mathrm{Fe}$ porphyrin is expected to yield the low-spin $\mathrm{S}=1 / 2$ complex $^{35}$ (Fig. 1d). The mechanism of NO binding to $\mathrm{Fe}$ porphyrin can be seen as an intermediate between the two previously discussed $\mathrm{d}^{7}$ and $\mathrm{d}^{5}$ complexes and is primarily reflected in a Fe-NO angle of $\sim 170^{\circ}$ for $\mathrm{FeP}-$ in between $120^{\circ}$

$35\left(d^{7}\right)$ and $180^{\circ}\left(d^{5}\right)$. Furthermore, the intermediate spin state $(S=1)$ of $\mathrm{Fe}(\mathrm{II})$ porphyrin may change its spin state easily, e.g. the histidine-bound natural Fe-porphyrin is high-spin $(\mathrm{S}=2)^{48}$.

It is worth to mention that from all three on-surface metalloporphyrins reacting with the NO-ligand, it is the Fe-porphyrin
40 which shows the best reversibility upon heating. NO is completely desorbed from the FeTPP on-surface complex at $\sim 260^{\circ} \mathrm{C}$ (Fig. S5 in the ESI $\dagger$ ) thus at lower temperature than CoTPP $\left(\sim 340^{\circ} \mathrm{C}\right)$ and in contrast to MnTPP which is found to be irreversibly bound within the experimental range up to $400^{\circ} \mathrm{C}$. 45 This reflects the distinctive bonding fashion and strength between $\mathrm{NO}$ and $\mathrm{Co}, \mathrm{Fe}$ and $\mathrm{Mn}$ ions.

Our DFT+U calculations (Fig. S7 in the ESI $\uparrow$ ) for FeP on the $\mathrm{Ni}$ surface have been performed for the molecule both being in physisorbed and chemisorbed conformation. The calculations 50 yield spin states between $\mathrm{S}=3 / 2$ and $\mathrm{S}=2$ in case of physisorption $\left(\sim 3.73 \mu_{\mathrm{B}}\right)$ and chemisorption $\left(\sim 3.57 \mu_{\mathrm{B}}\right)$. For the physisorbed and chemisorbed NO-FeP complex we find spin states of $\sim 1.93$ $\mu_{\mathrm{B}}$ and $\sim 3.49 \mu_{\mathrm{B}}$, respectively. On the basis of the observed reduction of the Fe-XMCD signal (Figs. 2a and 2b) and the 55 calculations suggesting that the exchange coupling strength is not reduced significantly (Fig. S6 in the ESI + ), we favor the physisorbed FeP configuration which shows a significant reduction of the spin state.

\section{On-surface magnetochemistry $\left(\mathrm{MnPc} / \mathrm{Co}+\mathrm{NH}_{3}\right)$ : Tuning the ${ }_{60}$ exchange coupling.}

We now discuss the on-surface magnetochemistry of the $\mathrm{MnPc} / \mathrm{Co}$ system $(\mathrm{S}=3 / 2)$ and the subsequent effect induced by $\sigma$ donating axial $\mathrm{NH}_{3}$ ligand. Without axial $\mathrm{NH}_{3}$ ligand, our calculations for chemisorbed $\mathrm{MnPc}$ on $\mathrm{Co}$ (Fig. 4) find a ${ }_{65}$ magnetic moment of $\sim 3.25 \mu_{\mathrm{B}}$ (spin state in between $\mathrm{S}=3 / 2$ and $\mathrm{S}=2$ ) and a strong hybridization between the surface and the outof plane d-orbitals $\left(\mathrm{d}_{\mathrm{z}}{ }^{2}\right.$ and $\left.\mathrm{d}_{\pi}\right)$. The calculations also yield a high coupling energy of $\sim 189 \mathrm{meV}$. Experimentally, this is reflected in the nearly constant $\mathrm{XMCD} / \mathrm{XAS}$ ratio as a function of 70 temperature (Fig. S4 in the ESI $\dagger$ ) which yields a lower limit for the exchange energy in the order of $\sim 103 \mathrm{meV}$ as discussed further in the Electronic Supplementary Information (ESI $\dagger$ ).

In the calculations for $\mathrm{MnPc} / \mathrm{Co}$ with axial $\mathrm{NH}_{3}$, the ligand pulls the Mn-ion out of the phthalocyanine plane by $\sim 0.42 \AA$ from 75 initially $2.30 \AA$ and leads to an increased spin of $\sim 4.45 \mu_{\mathrm{B}}$ (between $\mathrm{S}=2$ and $\mathrm{S}=5 / 2$ ). In the corresponding experimental data, the higher spin state is expressed by a higher XMCD/XAS ratio observed at lower temperatures (Figs. 2d1-d2). Remarkably, in our calculations the exchange coupling energy was found to be 80 reduced to only $\sim 4 \mathrm{meV}$. In the temperature dependent $\mathrm{XAS} / \mathrm{XMCD}$ data, the reduction of the exchange energy is reflected in a significant decrease of the relative XMCD signal with increasing temperature (Fig. S4 in the ESI $\dagger$ ). The data yield an accordingly reduced exchange energy of $\sim 31 \mathrm{meV}$. Notably, a 85 manipulation of the exchange energy has been claimed for $\mathrm{Fe}$ porphyrin, on the basis of the observed reduction of the circular dichroism after NO exposure ${ }^{49}$, but the system has not been measured at different temperatures. We also note that the calculations and experiments for FeTPP/Ni and NO-FeTPP/Ni do 90 not show significant differences in the exchange energy (Fig. S6 in the ESI $\dagger)$. The here presented $\mathrm{MnPc} / \mathrm{Co}+\mathrm{NH}_{3}$ case unambiguously demonstrates the relation between axial coordination and exchange energy, i.e. surface spin-trans effect.

\section{Conclusions}

95 The presented data provide insight into the on-surface 
magnetochemistry of spin-bearing square-planar complexes. The spins of the complexes (CoTPP, FeTPP, MnTPP and MnPc) are, before coordination with the optional axial ligand, found to be ferromagnetically coupled to the ferromagnetic substrate. The ${ }_{5} \mathrm{DFT}+\mathrm{U}$ calculations reveal a significant hybridization between the d-states in the transition-metal centers and the surface-ligand and show how the hybridization is affecting the spin states in the on-surface complexes. Subsequent axial coordination with the gaseous ligands ( $\mathrm{NO}$ or $\mathrm{NH}_{3}$ ) was found to lead i) to a 10 rearrangement of the electronic structure in general agreement with coordination chemistry and ii) to induce a structural trans effect on the surface with a magnitude depending on the exact chemical species involved. The structural trans effect is deduced from the calculated increase of the distance between the 15 transition-metal center and the substrate as well as the decrease of the hybridization with the surface-ligand. In the XMCD data of $d^{7}, d^{6}, d^{5}$ porphyrins and NO this is reflected respectively by a quenching of the spin in CoTPP/Ni, a modification of the spin in FeTPP/Ni, and most notably a remaining magnetic moment in $20 \mathrm{MnTPP} / \mathrm{Co}$ whose alignment with the substrate changed from FM to AFM by axial-ligation. The last system, namely $\mathrm{MnPc} / \mathrm{Co}$ demonstrates that an axial ligand (here $\mathrm{NH}_{3}$ ) may lead to a reduction of the exchange coupling strength. Hence, our data provide compelling evidence that on-surface axial-ligation leads 25 to a trans effect, which influences the molecular spin state as well as the sign and strength of the exchange interaction. We therefore propose the term surface spin-trans effect for the consequences of the structural trans effect on the spin and the exchange coupling's sign and strength.

30 The surface spin-trans effect is anticipated to serve as a powerful concept in the design of tailor-made spin-tunable metal-organic interfaces, which may find applications in magnetochemical sensors, in components for future spintronic devices or quantum computing building-blocks. Our study underlines that on-surface 35 magnetochemistry is emerging as a novel arena challenging the notions of classical coordination chemistry.

\section{Acknowledgements}

We gratefully acknowledge financial support from the Swiss National Science Foundation (SNSF), National Centre of ${ }_{40}$ Competence in Research Nanosciences (NCCR-Nano), Holcim Foundation for the Advancement of Scientific Research, Switzerland; and from the Swedish-Indian Research Links Programme and the C. Tryggers Foundation, Sweden. Part of this work has been performed at the SIM beamline of SLS, Paul ${ }_{45}$ Scherrer Institut, Switzerland. The authors sincerely thank Rolf Schelldorfer for technical support all throughout; Kees Landheer and Jan Nowakowski for help during beamtime experiments; and Md. Ehesan Ali for helpful discussions. N.B. thanks K. N. Ganesh (IISER Pune) for the support during beamtimes at SLS. ${ }_{50}$ Support from the Swedish National Infrastructure for Computing (SNIC) is also acknowledged.

\section{Notes and references}

1. K. M. Kadish, K. M. Smith, and R. Guilard, The Porphyrin Handbook, Academic Press, 1999.

55 2. J. V. Barth, Annu. Rev. Phys. Chem., 2007, 58, 375-407.
3. T. Kudernac, S. Lei, J. A. A. W. Elemans, and S. De Feyter, Chem. Soc. Rev., 2009, 38, 402.

4. L. Bartels, Nature Chemistry, 2010, 2, 87-95.

5. K. Seufert, M.-L. Bocquet, W. Auwärter, A. Weber-Bargioni, J. 60 Reichert, N. Lorente, and J. V. Barth, Nat. Chem., 2011, 3, 114-119.

6. K. Seufert, W. Auwärter, and J. V. Barth, J. Am. Chem. Soc., 2010, 132, 18141-18146.

7. B. J. Coe and S. J. Glenwright, Coordin. Chem. Rev., 2000, 203, 580 .

65 8. T. Lukasczyk, K. Flechtner, L. R. Merte, N. Jux, F. Maier, J. M. Gottfried, and H.-P. Steinruck, J. Phys. Chem. C, 2007, 111, 30903098 .

9. K. Flechtner, A. Kretschmann, H.-P. Steinrück, and J. M. Gottfried, J. Am. Chem. Soc., 2007, 129, 12110-12111.

70 10. W. Hieringer, K. Flechtner, A. Kretschmann, K. Seufert, W. Auwärter, J. V. Barth, A. Görling, H.-P. Steinrück, and J. M. Gottfried, J. Am. Chem. Soc., 2011, 133, 6206-6222.

11. C. Isvoranu, B. Wang, K. Schulte, E. Ataman, J. Knudsen, J. N. Andersen, M. L. Bocquet, and J. Schnadt, J. Phys.: Condens. Matter, 2010, 22, 472002.

12. A. Scheybal, T. Ramsvik, R. Bertschinger, M. Putero, F. Nolting, and T. A. Jung, Chem. Phys. Lett., 2005, 411, 214-220.

13. H. Wende, M. Bernien, J. Luo, C. Sorg, N. Ponpandian, J. Kurde, J. Miguel, M. Piantek, X. Xu, P. Eckhold, W. Kuch, K. Baberschke, P. M. Panchmatia, B. Sanyal, P. M. Oppeneer, and O. Eriksson, Nat. Mater., 2007, 6, 516-520.

14. M. Bernien, X. Xu, J. Miguel, M. Piantek, P. Eckhold, J. Luo, J. Kurde, W. Kuch, K. Baberschke, H. Wende, and P. Srivastava, Phys. Rev. B, 2007, 76, 214406.

85 15. P. M. Panchmatia, B. Sanyal, and P. Oppeneer, Chem. Phys., 2008, $343,47-60$.

16. M. Bernien, J. Miguel, C. Weis, M. E. Ali, J. Kurde, B. Krumme, P. M. Panchmatia, B. Sanyal, M. Piantek, P. Srivastava, K. Baberschke, P. M. Oppeneer, O. Eriksson, W. Kuch, and H. Wende, Phys. Rev. Lett., 2009, 102, 047202.

17. P. M. Oppeneer, P. M. Panchmatia, B. Sanyal, O. Eriksson, and M. E. Ali, Prog. Surf. Sci., 2009, 84, 18-29.

18. D. Chylarecka, C. Wäckerlin, T. K. Kim, K. Müller, F. Nolting, A. Kleibert, N. Ballav, and T. A. Jung, J. Phys. Chem. Lett., 2010, 1, $1408-1413$.

19. D. Chylarecka, T. K. Kim, K. Tarafder, K. Müller, K. Gödel, I. Czekaj, C. Wäckerlin, M. Cinchetti, M. E. Ali, C. Piamonteze, F. Schmitt, J.-P. Wüstenberg, C. Ziegler, F. Nolting, M. Aeschlimann, P. M. Oppeneer, N. Ballav, and T. A. Jung, J. Phys. Chem. C, 2011, 115, 1295-1301.

20. A. Lodi Rizzini, C. Krull, T. Balashov, J. J. Kavich, A. Mugarza, P. S. Miedema, P. K. Thakur, V. Sessi, S. Klyatskaya, M. Ruben, S. Stepanow, and P. Gambardella, Phys. Rev. Lett., 2011, 107, 177205.

21. J. Brede, N. Atodiresei, S. Kuck, P. Lazić, V. Caciuc, Y. Morikawa, 105 G. Hoffmann, S. Blügel, and R. Wiesendanger, Phys. Rev. Lett., 2010, 105, 047204.

22. S. Stepanow, P. Miedema, A. Mugarza, G. Ceballos, P. Moras, J. Cezar, C. Carbone, F. de Groot, and P. Gambardella, Phys. Rev. B, 2011, 83, 220401.

110 23. N. Tsukahara, K. Noto, M. Ohara, S. Shiraki, N. Takagi, S. Shin, and M. Kawai, Phys. Rev. Lett., 2009, 102, 167203.

24. S. Stepanow, A. Mugarza, G. Ceballos, P. Moras, J. C. Cezar, C. Carbone, and P. Gambardella, Phys. Rev. B, 2010, 82, 014405.

25. S. Lach, A. Altenhof, K. Tarafder, F. Schmitt, M. E. Ali, M. Vogel, J. 115 Sauther, P. M. Oppeneer, and C. Ziegler, Adv. Funct. Mater., 2012, 22, 989-997.

26. A. Mugarza, C. Krull, R. Robles, S. Stepanow, G. Ceballos, and P. Gambardella, Nat Commun., 2011, 2, 490.

27. C. Wäckerlin, D. Chylarecka, A. Kleibert, K. Müller, C. Iacovita, F. 120 Nolting, T. A. Jung, and N. Ballav, Nat. Commun., 2010, 1, 61.

28. S. Javaid, M. Bowen, S. Boukari, L. Joly, J.-B. Beaufrand, X. Chen, Y. Dappe, F. Scheurer, J.-P. Kappler, J. Arabski, W. Wulfhekel, M. Alouani, and E. Beaurepaire, Phys. Rev. Lett., 2010, 105, 077201.

29. H. C. Siegmann and J. Stöhr, Magnetism: From Fundamentals to $125 \quad$ Nanoscale Dynamics, Springer, Berlin, 1st ed., 2006. 
30. M. E. Ali, B. Sanyal, and P. M. Oppeneer, J. Phys. Chem. B, 2012, 116, 5849-5859.

31. M. E. Ali, B. Sanyal, and P. M. Oppeneer, J. Phys. Chem. C, 2009, 113, 14381-14383.

5 32. H. Goff, G. N. La Mar, and C. A. Reed, J. Am. Chem. Soc., 1977, 99, 3641-3646.

33. L. J. Boucher, J. Am. Chem. Soc., 1970, 92, 2725-2730.

34. B. B. Wayland and J. V. Minkiewicz, J. Chem. Soc., Chem. Commun., 1976, 1015.

10 35. B. B. Wayland and L. W. Olson, J. Am. Chem. Soc., 1974, 96, 60376041.

36. B. B. Wayland, L. W. Olson, and Z. U. Siddiqui, J. Am. Chem. Soc., 1976, 98, 94-98.

37. J. Huheey, Inorganic chemistry: principles of structure and

15 reactivity, HarperCollins College Publishers, New York, 4th ed., 1993.

38. W. Kaim and B. Schwederski, Coordin. Chem. Rev., 2010, 254, $1580-1588$.

39. T. Kataoka, Y. Sakamoto, Y. Yamazaki, V. R. Singh, A. Fujimori, Y.

20 Takeda, T. Ohkochi, S.-I. Fujimori, T. Okane, Y. Saitoh, H. Yamagami, and A. Tanaka, Solid State Commun., 2012, 152, 806809.

40. A. Zhao, Q. Li, L. Chen, H. Xiang, W. Wang, S. Pan, B. Wang, X. Xiao, J. Yang, J. G. Hou, and Q. Zhu, Science, 2005, 309, 15421544.

41. C. Iacovita, M. Rastei, B. Heinrich, T. Brumme, J. Kortus, L. Limot, and J. Bucher, Phys. Rev. Lett., 2008, 101, 116602.

42. X. Chen, Y.-S. Fu, S.-H. Ji, T. Zhang, P. Cheng, X.-C. Ma, X.-L. Zou, W.-H. Duan, J.-F. Jia, and Q.-K. Xue, Phys. Rev. Lett., 2008, 101, 197208.

43. E. Annese, J. Fujii, I. Vobornik, G. Panaccione, and G. Rossi, Phys. Rev. B, 2011, 84, 174443.

44. G. R. A. Wyllie and W. R. Scheidt, Chem. Rev., 2002, 102, 10671090.

35 45. C. G. Barraclough, R. L. Martin, S. Mitra, and R. C. Sherwood, J. Chem. Phys., 1970, 53, 1638.

46. W. R. Scheidt and J. L. Hoard, J. Am. Chem. Soc., 1973, 95, 82818288.

47. W. R. Scheidt, K. Hatano, G. A. Rupprecht, and P. L. Piciulo, Inorg.

40 Chem., 1979, 18, 292-299.

48. J. M. Friedman, T. W. Scott, R. A. Stepnoski, M. Ikeda-Saito, and T. Yonetani, J. Biol. Chem., 1983, 258, $10564-10572$.

49. J. Miguel, C. F. Hermanns, M. Bernien, A. Krüger, and W. Kuch, J. Phys. Chem. Lett., 2011, 2, 1455-1459.

45 